\title{
Non-accessible Critical Points of Cremer Polynomials
}

\author{
Jan Kiwi \\ Mathematics Deparment \\ S.U.N.Y. Stony Brook
}

Abstract

It is shown that a polynomial with a Cremer periodic point has a non-accessible critical point in its Julia set provided that the Cremer periodic point is approximated by small cycles.

\section{Introduction.}

Cremer fixed points occur as fixed points of polynomial maps from the complex plane onto itself. Their presence forces the Julia set not to be locally connected [S1] [D. When local connectivity fails it is of interest to determine which points in the Julia set are accessible from the complement of the Julia set and which are not (if any).

Repelling periodic points are always accessible [H] [E] and it 'often' happens that Cremer fixed points are 'surrounded' by repelling cycles. Let us be more precise, if every neighborhood of a Cremer fixed point contains infinitely many repelling cycles, we say that the Cremer fixed point is approximated by small cycles. For quadratic polynomials, J.-C. Yoccoz has shown that this is always the case [Y]. For polynomials of higher degree it is an open problem. Some results and a more detailed discussion about this problem can be found in [M2] and [PM1].

In this paper we show how the accessibility of the small cycles implies the non-accessibility of a critical point.

Theorem 1.1 Let $P$ be a polynomial with a Cremer fixed point $\hat{z}$ that is approximated by small cycles. Then there exists a critical point which is not accessible from the complement of the Julia set.

We conclude that the critical point of a quadratic polynomial with a Cremer periodic point is not accessible, thus answering a question posed by J. Milnor in BL.

Corollary 1.2 The critical point of a quadratic polynomial with a Cremer periodic point is not accessible from the complement of the Julia set.

Under the assumption that the Julia set is connected, each point that is accessible from the complement of the filled Julia set is the landing point of some external ray. In this case, we prove a stronger statement by showing the existence of a non-accessible critical value with the following property. An external ray (if any) that accumulates at this critical value also accumulates at the Cremer fixed point. 
Theorem 1.3 Let $P$ be a polynomial with connected Julia set $J_{P}$ and with a Cremer fixed point $\hat{z}$ that is approximated by small cycles. Then there exists a critical value $v$ which is not accessible from $\mathbb{C} \backslash J_{P}$ such that: if $v$ belongs to the closure $\bar{R}$ of some external ray $R$, then $\hat{z}$ also belongs to $\bar{R}$.

For some quadratic polynomials our result can be deduced from a stronger one of A. Douady (compare $[\mathrm{S} \emptyset]$ ). More precisely, for a generic quadratic polynomial with a Cremer fixed point, Douady showed that there is an external ray that does not land; furthermore, this external ray accumulates in a set containing the Cremer fixed point and its preimage. It follows that for such quadratic polynomials every external ray which accumulates at the critical value also accumulates at the Cremer fixed point (see Corollary 3.5, Section 3).

Recently, R. Pérez-Marco [PM2] has shown that rational functions with Cremer periodic points always have some non-accessible points in their Julia set. For a 'large' family of infinitely renormalizable quadratic polynomials, he proves the non-accessibility of the critical point; and for a generic quadratic polynomial with a Cremer fixed point, Pérez-Marco shows that the critical point is not accessible.

This paper is organized as follows:

In Section 2, we briefly summarize some definitions and results from polynomial dynamics. For general background in complex dynamics see [M1] and [L].

In Section 3, we prove some preliminary results. Also, we show how one of them leads to a new proof (for polynomials with connected Julia set) of a well known result by A. Douady [B] and M. Shishikura [Sh]. That is, for a polynomial of degree $d$, the total number of Cremer cycles and cycles of bounded Fatou components is strictly less than d (Corollary 3.4).

In Section 4, we prove our main results. The proof of Theorem 1.3 is by contradiction and the idea is a refinement of the following line of argument. Under the assumption that certain critical values are accessible, we construct an open region around the Cremer fixed point containing at most finitely many cycles. This region is obtained by cutting the complex plane along an appropriate collection of external rays. The correct choice of these external rays is based on the results from Section 3. In order to prove Theorem 1.1 in the disconnected case we use some results about polynomial like mappings and then we apply Theorem 1.3 to find a non-accessible critical point.

Acknowledgments: I am grateful to John Milnor for many interesting and inspiring discussions. I would like to thank Misha Lyubich and Alfredo Poirier for many helpful conversations. The author is partially supported by a "Beca Presidente de la Republica" (Chile).

\section{Cremer Points and Accessible Points.}

Consider a polynomial $P: \mathbb{C} \rightarrow \mathbb{C}$ of degree $d \geq 2$ with a fixed point $\hat{z}$ of multiplier $\lambda=P^{\prime}(\hat{z})$. The fixed point is called repelling if $|\lambda|>1$, attracting if $|\lambda|<1$ and parabolic if $\lambda$ is a root of unity.

For $\lambda=e^{2 \pi i \theta}$ where $\theta$ is irrational, we have to distinguish between two possibilities. If the fixed point $\hat{z}$ belongs to the Julia set $J_{P}$ we say that $\hat{z}$ is a Cremer fixed point. Otherwise, if $\hat{z}$ lies in the Fatou set $F_{P}$, call $\hat{z}$ a Siegel fixed point.

For a generic choice of $\theta, \mathrm{H}$. Cremer $\mathrm{Cr}$ showed that there exists a sequence of periodic points converging to $\hat{z}$, in this case $\hat{z}$ belongs to the Julia set. In contrast, for a full Lebesgue measure 
set of angles $\theta$, C.L. Siegel [Si] proved that $\hat{z}$ lies in the Fatou set. We refer the reader to [M2] and PM1 for a more detailed exposition about this dichotomy.

A modification of Cremer's proof (compare M2]) shows that for a generic choice of $\theta$, every neighborhood of a Cremer fixed point $\hat{z}$ contains infinitely many cycles. We say that $\hat{z}$ is approximated by small cycles. According to Yoccoz $[\mathrm{Y}]$ quadratic Cremer fixed points are always approximated by small cycles. It is not known whether this is true for polynomials of arbitrary degree. For some interesting results about this problem see PM1.

A point $z$ in the Julia set $J_{P}$ is called accessible from a Fatou component $V$ if there exists a path $\gamma$ contained in $V$ that ends at $z$, i.e.

$$
\gamma:[0,1) \rightarrow V
$$

and $\gamma(r) \rightarrow z$ as $r \rightarrow 1$. Accessibility is a property completely invariant under the dynamics, $z$ is accessible from $V$ if and only if $P(z)$ is accessible from $P(V)$. When $z$ is not accessible from any Fatou component sometimes we just say that $z$ is a non-accessible point.

Let us now assume that $K_{P}$ is connected. For simplicity we also assume that $P$ is a monic polynomial. The complement of the closed unit disc $\bar{\Delta}$ is conformally isomorphic to the complement of $K_{P}$. Moreover, there exists a unique conformal isomorphism:

$$
\phi: \mathbb{C} \backslash \bar{\Delta} \rightarrow \mathbb{C} \backslash K_{P}
$$

such that

$$
P \circ \phi(z)=\phi\left(z^{d}\right)
$$

and that is asymptotic to identity near infinity (see M1]).

The question of which points in $J_{P}$ are accessible from $\mathbb{C} \backslash K_{P}$ is closely related to the boundary behavior of the map $\phi$. We say that

$$
R_{t}=\phi\left(\left\{r e^{2 \pi i t}: r \in(1, \infty)\right\}\right)
$$

is the external ray with angle $t \in \mathbb{R} / \mathbb{Z}$. An external ray $R_{t}$ lands at $z$ if $\phi\left(r e^{2 \pi i t}\right) \rightarrow z$ as $r \rightarrow 1$. Therefore, the landing points of external rays are accessible from $\mathbb{C} \backslash K_{P}$. A result of Lindelöf assures that the converse is also true, every point accessible from $\mathbb{C} \backslash K_{P}$ is the landing point of some external ray (compare $\mathrm{Ru}$ ).

The external ray $R_{t}$ is called a periodic ray if $P^{\circ n}\left(R_{t}\right)=R_{t}$ for some positive integer $n$. In particular, if $P\left(R_{t}\right)=R_{t}$ we say that the external ray $R_{t}$ is a fixed ray. Periodic rays always land, moreover their landing points are parabolic or repelling periodic points. Conversely, parabolic and repelling periodic points are the landing points of at least one periodic ray. (see [H] [M1])

If an external ray $R$ lands, then the closure $\bar{R}$ is the union of the external ray and its landing point. Otherwise, if $R$ fails to land, $\bar{R}$ is the union of the external ray and a non-trivial connected subset of the Julia set. For short, the closure $\bar{R}$ of an external ray $R$ is called a closed ray.

\section{Separation Lemmas.}

For polynomials with connected Julia sets, A. Poirier pointed out that the union of all closed fixed rays 'separates' invariant Fatou components and Cremer fixed points. This is stated as a corollary of 
a result by L. Goldberg and J. Milnor (compare with Theorem 3.3 and Corollary 3.5 in GM). We go one step further and show that after cutting the complex plane along an appropriate collection of external rays, each Cremer periodic point and periodic Fatou component is 'separated' from the others:

Lemma 3.1 Let $P$ be a polynomial with connected Julia set. Then there exists a finite union $\mathcal{C}$ of closed periodic rays such that:

$P(\mathcal{C})=\mathcal{C}$.

Each component of $\mathbb{C} \backslash \mathcal{C}$ contains at most one Cremer periodic point or periodic Fatou component.

Recall that periodic rays always land, hence the corresponding closed ray is the union of the periodic ray and its landing point. In this section, the closed rays taken into consideration are always the closure of external rays that land.

Lemma 3.1 is an immediate consequence of the following (compare with Theorem 3.3 in GM]):

Theorem 3.2 (Goldberg and Milnor) Let $P$ be a polynomial with connected Julia set. Denote by $\mathcal{C}$ the union of all closed fixed rays:

$$
\mathcal{C}=\bar{R}_{0} \cup \bar{R}_{1 / d-1} \cup \cdots \cup \bar{R}_{d-2 / d-1}
$$

If $U$ is a connected component of $\mathbb{C} \backslash \mathcal{C}$, then one and only one of the following holds:

$U$ contains exactly one invariant Fatou component.

$U$ contains exactly one fixed point which belongs to the Julia set. 
Proof of Lemma 3.1: If $P$ has no Cremer periodic points and no bounded periodic Fatou components there is nothing to prove. Otherwise, let $m$ be the minimum common multiple of the following list of integers: periods of periodic Fatou components, and periods of Cremer periodic points. The number of non-repelling cycles is finite, hence this list is also finite and $m$ is a well defined integer.

Periodic Fatou components and Cremer periodic points of $P$ and $P^{\circ m}$ are the same, only their periods differ. In fact, we have chosen $m$ in such a way that the periodic Fatou components are invariant under $P^{\circ m}$ and that the Cremer periodic points are fixed points in its Julia set.

Now consider the union $\mathcal{C}$ of closed rays fixed by $P^{\circ m}$ :

$$
\mathcal{C}=\overline{R_{0}} \cup \cdots \cup \bar{R}_{d^{m}-2 / d^{m}-1} .
$$

Since fixed rays land at parabolic or repelling points, this set $\mathcal{C}$ is disjoint from bounded Fatou components and Cremer points. Applying the previous Theorem to $P^{\circ m}$, it follows that the set $\mathcal{C}$ has the desired properties.

Let us introduce some notation. If $\mathcal{C}$ is the union of finitely many closed external rays and $z$ is a point that lies in the complement of $\mathcal{C}$, denote by $U_{\mathcal{C}}(z)$ the connected component of $\mathbb{C} \backslash \mathcal{C}$ that contains $z$. In a similar way, if $V$ is a connected set contained in the complement of $\mathcal{C}$, denote by $U_{\mathcal{C}}(V)$ the connected component of $\mathbb{C} \backslash \mathcal{C}$ that contains $V$.

A refinement of the previous Lemma shows that cutting along a larger collection of external rays, critical points and Cremer points that are left 'together', stay 'together' under forward iterations. The corresponding statement for periodic Fatou components also holds.

Lemma 3.3 Let $P$ be a polynomial with connected Julia set. Then there exists a finite union $\mathcal{C}$ of closed rays such that:

(1) $P(\mathcal{C}) \subset \mathcal{C}$.

(2) Each component of $\mathbb{C}-\mathcal{C}$ contains at most one Cremer periodic point or periodic Fatou component.

(3) If $\hat{z}$ is a Cremer periodic point and $c$ is a critical point such that $U_{\mathcal{C}}(c)=U_{\mathcal{C}}(\hat{z})$, then $U_{\mathcal{C}}\left(P^{\circ n}(c)\right)=U_{\mathcal{C}}\left(P^{\circ n}(\hat{z})\right)$ for all $n \geq 1$. Moreover, $c \in J_{P}$ and $c$ does not belong to the closure of any bounded Fatou component.

(4) If $V$ is a bounded periodic Fatou component and $c$ is a critical point such that $U_{\mathcal{C}}(c)=U_{\mathcal{C}}(V)$, then $U_{\mathcal{C}}\left(P^{\circ n}(c)\right)=U_{\mathcal{C}}\left(P^{\circ n}(V)\right)$ for all $n \geq 1$.

Remark: The proof of Corollary 3.4 will show that statements (3) and (4) of the previous Lemma are not vacuous in the following sense. Given a Cremer periodic orbit $z_{0}=P\left(z_{n-1}\right), \ldots, z_{n-1}=$ $P\left(z_{n-2}\right)$, then for some $z_{i}$ there exists a critical point $c$ such that $U_{\mathcal{C}}(c)=U_{\mathcal{C}}\left(z_{i}\right)$. The corresponding assertion is also true for bounded periodic Fatou components.

Proof: Let $\mathcal{C}_{0}$ be the union of closed rays from Lemma 3.1. For $k \geq 1$ define inductively $\mathcal{C}_{k}=$ $P^{-1}\left(\mathcal{C}_{k-1}\right)$.

We are going to show that conditions (1) through (4) hold for $\mathcal{C}_{k}$ when $k$ is large enough. Before let us list some properties of the sets $\mathcal{C}_{k}$ and their complementary regions:

(a) $\mathcal{C}_{k} \subset \mathcal{C}_{k+1}$ for $k \geq 0$

(b) $P\left(\mathcal{C}_{k}\right) \subset \mathcal{C}_{k}$ for $k \geq 0$,

(c) $U_{\mathcal{C}_{k+1}}(z) \subset U_{\mathcal{C}_{k}}(z)$ for $k \geq 0$ and $z \in \mathbb{C}-\mathcal{C}_{k+1}$, 
(d) $P^{\circ n}\left(U_{\mathcal{C}_{k+n}}(z)\right)=U_{\mathcal{C}_{k}}\left(P^{\circ n}(z)\right)$ for $k \geq 0, n \geq 1$ and $z \in \mathbb{C}-\mathcal{C}_{k+n}$.

All of the above can be deduced from the forward invariance of $\mathcal{C}_{0}\left(P\left(\mathcal{C}_{0}\right)=\mathcal{C}_{0}\right)$.

From Lemma 3.1 and properties (b) and (c) it follows that conditions (1) and (2) hold for all $\mathcal{C}_{k}$.

Claim 1: Given a critical point $c$ and a Cremer periodic point $\hat{z}$ there exists $k(c, \hat{z}) \geq 0$ such that:

$(*)$ if $U_{\mathcal{C}_{l}}(c)=U_{\mathcal{C}_{l}}(\hat{z})$, then $U_{\mathcal{C}_{l}}\left(P^{\circ n}(c)\right)=U_{\mathcal{C}_{l}}\left(P^{\circ n}(\hat{z})\right)$ for all $n \geq 1$;

holds for all $l \geq k(c, \hat{z})$.

Proof of Claim 1: In the case that $(*)$ holds for all $l \geq 0$, of course we take $k(c, \hat{z})=0$. Otherwise, for some $l_{0} \geq 0$ we have that $U_{\mathcal{C}_{l_{0}}}(c)=U_{\mathcal{C}_{l_{0}}}(\hat{z})$ and $U_{\mathcal{C}_{l_{0}}}\left(P^{\circ n}(c)\right) \neq U_{\mathcal{C}_{l_{0}}}\left(P^{\circ n}(\hat{z})\right)$ for some $n \geq 1$. In this case let $k(c, \hat{z})=l_{0}+n$, it follows from (c) and (d) that $U_{\mathcal{C}_{l}}(c) \neq U_{\mathcal{C}_{l}}(\hat{z})$ for all $l \geq k(c, \hat{z})$.

We omit the identical proof of the corresponding claim for periodic Fatou components:

Claim 2: Given a critical point $c$ and a bounded periodic Fatou component $V$ there exists $k(c, V) \geq 0$ such that:

$(*)$ if $U_{\mathcal{C}_{l}}(c)=U_{\mathcal{C}_{l}}(V)$, then $U_{\mathcal{C}_{l}}\left(P^{\circ n}(c)\right)=U_{\mathcal{C}_{l}}\left(P^{\circ n}(V)\right)$ for all $n \geq 1$;

holds for all $l \geq k(c, V)$.

Let $N$ be the maximum of the numbers $k(c, \hat{z})$ and $k(c, V)$, where all possible pairs $(c, \hat{z})$ and $(c, V)$ are taken into consideration. Since the number of critical points and non-repelling cycles is finite, the total number of all such pairs is also finite and $N$ is a well defined integer.

As mentioned before Claim $1, \mathcal{C}_{N}$ satisfies conditions (1) and (2). From Claim 2, it follows that (4) holds for $\mathcal{C}_{N}$. It remains to complete the proof that (3) also holds for $\mathcal{C}_{N}$.

If $c$ is a critical point and $\hat{z}$ is a Cremer point such that $U_{\mathcal{C}_{N}}(c)=U_{\mathcal{C}_{N}}(\hat{z})$, then

$$
U_{\mathcal{C}_{N}}\left(P^{\circ n}(c)\right)=U_{\mathcal{C}_{N}}\left(P^{\circ n}(\hat{z})\right) \neq U_{\mathcal{C}_{N}}(V)
$$

for all $n \geq 1$ and for all bounded periodic Fatou components $V$. According to D. Sullivan [S2], every point in the closure of a bounded Fatou component is mapped after a finite number of iterates to the closure of a bounded periodic Fatou component. It follows that $c$ belongs to $J_{P}$ and $c$ does not belong to the closure of any bounded Fatou component.

According to Douady [B]] and Shishikura [Sh], the total number of Cremer cycles and cycles of bounded Fatou components of $P$ is strictly less than $d=\operatorname{deg} P$. For polynomials with connected Julia sets, Lemma 3.1 leads to a new proof of this result.

Corollary 3.4 Let $P$ be a polynomial with connected Julia set. Then the total number of Cremer cycles and cycles of bounded Fatou components is less than or equal to the number of distinct critical points.

Proof: The idea is to show that to each Cremer cycle corresponds at least one critical point and to each cycle of bounded Fatou components also corresponds at least one critical point.

Apply Lemma 3.1 to obtain a finite union $\mathcal{C}$ of closed external rays. Consider a Cremer cycle $z_{0}=P\left(z_{n-1}\right), \ldots, z_{n-1}=P\left(z_{n-2}\right)$. We claim that there exists at least one critical point in $U_{\mathcal{C}}\left(z_{0}\right) \cup$ $\cdots \cup U_{\mathcal{C}}\left(z_{n-1}\right)$. In order to prove this claim let $\mathcal{C}_{1}=P^{-1}(\mathcal{C})$ and for $k \geq 2$ define inductively 
$\mathcal{C}_{k}=P^{-1}\left(\mathcal{C}_{k-1}\right)$. Suppose that $U_{\mathcal{C}}\left(z_{0}\right) \cup \cdots \cup U_{\mathcal{C}}\left(z_{n-1}\right)$ contains no critical points. In particular, $U_{\mathcal{C}_{n}}\left(z_{0}\right) \cup \cdots \cup U_{\mathcal{C}_{1}}\left(z_{n-1}\right)$ contains no critical point. It follows that the proper holomorphic map

$$
\left.P^{\circ n}\right|_{U_{\mathcal{C}_{n}}\left(z_{0}\right)}: U_{\mathcal{C}_{n}}\left(z_{0}\right) \rightarrow U_{\mathcal{C}}\left(z_{0}\right)
$$

has no critical points. Therefore, $\left.P^{\circ n}\right|_{U_{\mathcal{C}_{n}}\left(z_{0}\right)}$ is a conformal isomorphism of simply connected domains. By Schwartz Lemma, $U_{\mathcal{C}_{n}}\left(z_{0}\right)=U_{\mathcal{C}}\left(z_{0}\right)$ and $\left.P^{\circ n}\right|_{U_{\mathcal{C}_{n}}\left(z_{0}\right)}$ is conformally conjugate to a rotation of the unit disc, which contradicts the fact that $K_{P}$ is bounded. Hence, $U_{\mathcal{C}}\left(z_{0}\right) \cup \cdots \cup$ $U_{\mathcal{C}}\left(z_{n-1}\right)$ contains at least one critical point.

For a cycle $V_{0}=P\left(V_{n-1}\right), \ldots, V_{n-1}=P\left(V_{n-2}\right)$ of attracting or parabolic basins it is well known that $U_{\mathcal{C}}\left(V_{0}\right) \cup \cdots \cup U_{\mathcal{C}}\left(V_{n-1}\right)$ contains at least one critical point (see M1]). For a cycle of Siegel discs, an identical argument then the one used for Cremer cycles shows that $U_{\mathcal{C}}\left(V_{0}\right) \cup \cdots \cup U_{\mathcal{C}}\left(V_{n-1}\right)$ contains at least one critical point. The proof follows from the classification of periodic Fatou components.

In order to prove the remark after Lemma 3.3, repeat the proof of the previous Corollary but this time start with the finite union $\mathcal{C}$ of closed rays obtained from Lemma 3.3.

The next result shows how the existence of an external ray that accumulates at a Cremer fixed point and one of its preimages implies the existence of a non-accessible critical value. Moreover, the non-accessible critical value has the property that if an external ray accumulates at it, this external ray also accumulates at the Cremer fixed point.

Corollary 3.5 Let $P$ be a polynomial with connected $K_{P}$ and with a Cremer fixed point $\hat{z}$. If there exists an external ray $R_{0}$ such that $\{\hat{z}, z\} \subset \overline{R_{0}}$ for some $z \in P^{-1}(\hat{z}) \backslash\{\hat{z}\}$, then there exists a non-accessible critical value $v$ with the following property: every external ray $R$ that accumulates at $v$ also accumulates at $\hat{z}$.

Outline of the Proof: Apply Lemma 3.3 to obtain a finite union $\mathcal{C}$ of closed rays. Let $\mathcal{C}_{1}=P^{-1}(\mathcal{C})$ and notice that $R_{0} \subset U_{\mathcal{C}_{1}}(\hat{z})$. Call $c_{1}, \ldots, c_{k}$ the critical points that are contained in $U_{\mathcal{C}_{1}}(\hat{z})$. It follows that their critical values $v_{1}, \ldots, v_{k}$ are contained in $U_{\mathcal{C}}(\hat{z})$, and the map

$$
P: U_{\mathcal{C}_{1}}(\hat{z}) \backslash P^{-1}\left(\left\{v_{1}, \ldots, v_{k}\right\}\right) \rightarrow U_{\mathcal{C}}(\hat{z}) \backslash\left\{v_{1}, \ldots, v_{k}\right\}
$$

is a regular covering.

Take a small disc $D$ centered at $\hat{z}$. Call $D_{0}$ the preimage of $D$ that contains $\hat{z}$ and denote by $D_{1}, \ldots, D_{l}$ the other preimages of $D$ contained in $U_{\mathcal{C}_{1}}(\hat{z})$. It follows that there exists a closed segment $\alpha$ of $R_{0}$ contained in $U_{\mathcal{C}_{1}}(\hat{z}) \backslash D_{0} \cup \cdots \cup D_{l}$ such that $\alpha$ joins the boundary $\partial D_{0}$ of $D_{0}$ with the boundary $\partial D_{i}$ of $D_{i}$ for some $i \neq 0$. Also, we can assume that $\alpha$ intersects $\partial D_{0} \cup \partial D_{i}$ in exactly 2 points, which are the endpoints of $\alpha$.

Observe that $P(\alpha)$ is contained in $U_{\mathcal{C}}(\hat{z}) \backslash D$ and intersects $\partial D$ in exactly 2 points. The set $\partial D \cup P(\alpha)$ separates the plane into 3 components. That is, an unbounded component, the disc $D$, and a bounded component $\tilde{D}$. Call $\beta$ the boundary of the Jordan domain $\tilde{D} \subset U_{\mathcal{C}}(\hat{z})$. The curve $\beta$ is the union of a segment of $\partial D$ with $P(\alpha)$. Since a lift of $\beta$ is not a closed curve, $\tilde{D}$ contains a critical value $v$. By Lemma 3.3 (3) the critical value $v$ does not belong to the closure of any bounded Fatou component. If an external ray $R$ accumulates at $v$, then $R$ intersects $\partial D$. Taking $D$ arbitrarily small, it follows that $v$ has the desired properties. 


\section{Non-accessible Points.}

In this section first we prove the following:

Theorem 1.3 Let $P$ be a polynomial with connected Julia set $J_{P}$ and with a Cremer fixed point $\hat{z}$ that is approximated by small cycles. Then there exists a critical value $v$ which is not accessible from $\mathbb{C} \backslash J_{P}$ such that: if $v \in \bar{R}$ for some external ray $R$, then $\hat{z} \in \bar{R}$.

Proof of Theorem 1.3: Start applying Lemma 3.3 to obtain a finite union $\mathcal{C}$ of closed rays. Notice that all the critical points $c_{1}, \ldots, c_{k}$ contained in $U_{\mathcal{C}}(\hat{z})$ belong to the Julia set $J_{P}$. Thus, their critical values $v_{1}=P\left(c_{1}\right), \ldots, v_{k}=P\left(c_{k}\right)$ are also in $J_{P}$. These critical values are not in the closure of any bounded Fatou component. Therefore, it is enough to show that one of them is not accessible from $\mathbb{C} \backslash K_{P}$ and that every external ray which accumulates at this critical value also accumulates at the Cremer fixed point.

Let us first consider the case in which the Cremer fixed point is not a critical value. The proof is by contradiction. Assume that there exists external rays $R_{t_{1}}, \ldots, R_{t_{k}}$ such that $v_{i} \in \overline{R_{t_{i}}}$ and $\hat{z} \notin \overline{R_{t_{i}}}$. Consider the sets formed by the union of finitely many closed rays:

$$
\begin{gathered}
\mathcal{C}_{0}=\mathcal{C} \cup \overline{R_{t_{1}}} \cup \cdots \cup \overline{R_{t_{k}}}, \\
\mathcal{C}_{1}=P^{-1}\left(\mathcal{C}_{0}\right) ;
\end{gathered}
$$

and the corresponding simply connected domains containing the Cremer fixed point $\hat{z}$ :

$$
\begin{aligned}
& U_{0}=U_{\mathcal{C}_{0}}(\hat{z}), \\
& U_{1}=U_{\mathcal{C}_{1}}(\hat{z}) .
\end{aligned}
$$

The rest of the proof consists in showing that $U_{1}$ contains at most finitely many cycles. For each repelling cycle contained in $U_{1}$ there is at least one cycle of external rays contained in $U_{1}$. Then, it is enough to show that $U_{1}$ contains at most finitely many cycles of external rays.

First observe that the proper holomorphic map

$$
\left.P\right|_{U_{1}}: U_{1} \rightarrow U_{0}
$$

has no critical points. Regular coverings of simply connected domains are homeomorphisms. Therefore, $\left.P\right|_{U_{1}}$ is a conformal isomorphism.

The polynomial $P$ acts in the angles of the external rays as the multiplication by $d=\operatorname{deg} P$ map,

$$
\begin{aligned}
m_{d}: \mathbb{R} / \mathbb{Z} & \rightarrow \mathbb{R} / \mathbb{Z} \\
t & \mapsto d \cdot t
\end{aligned}
$$

We are interested in the external rays contained in $U_{1}$ and their image, the external rays contained in $U_{0}$. Denote by $B_{1}$ and $B_{0}$ the corresponding sets formed by the angles of such rays:

$$
\begin{aligned}
& B_{0}=\left\{t: R_{t} \subset U_{0}\right\} \subset \mathbb{R} / \mathbb{Z}, \\
& B_{1}=\left\{t: R_{t} \subset U_{1}\right\} \subset \mathbb{R} / \mathbb{Z} .
\end{aligned}
$$

As mentioned before the map $\left.P\right|_{U_{1}}: U_{1} \rightarrow U_{0}$ is a conformal isomorphism, therefore

$$
\left.m_{d}\right|_{B_{1}}: B_{1} \rightarrow B_{0}
$$


is a cyclic order preserving homeomorphism. Hence, $\left.m_{d}\right|_{B_{1}}$ admits a continuos extension $g: \mathbb{R} / \mathbb{Z} \rightarrow$ $\mathbb{R} / \mathbb{Z}$ which is a non-decreasing circle map (i.e. the lift is non-decreasing).

A non-decreasing circle map $g$ either is free of periodic orbits or all periodic orbits have the same period. If $m_{d}$ has cycles contained in $B_{1}$, then all of them have the same period. The map $m_{d}: \mathbb{R} / \mathbb{Z} \rightarrow \mathbb{R} / \mathbb{Z}$ has finitely many cycles of the same period. We conclude that $m_{d}$ has at most finitely many cycles contained in $B_{1}$. An equivalent statement is that there are at most finitely many cycles of external rays contained in $U_{1}$, which contradicts the assumption that the Cremer fixed point $\hat{z}$ is approximated by small cycles.

Under that assumption that $\hat{z}$ is not a critical value, we have proved that there exists a critical value $v \neq \hat{z}$ such that every ray that accumulates at $v$ also accumulates at $\hat{z}$. It follows that $v$ is not accessible from $\mathbb{C} \backslash K_{P}$

In the case that $\hat{z}$ is a critical value we outline how to modify the arguments used before. Call $c_{1}, \ldots, c_{k}$ the critical points of $P$ contained in $U_{\mathcal{C}}(\hat{z})$. List without repetition their critical values $v_{0}=\hat{z}, v_{1}, \ldots, v_{l}$. The proof also proceeds by contradiction. This time, we assume that $R_{t_{0}}$ lands at $v_{0}$ and that $v_{i} \in \overline{R_{t_{i}}}$ and $\hat{z} \notin \overline{R_{t_{i}}}$.

As before, consider the sets formed by the union of closed rays:

$$
\begin{gathered}
\mathcal{C}_{0}=\mathcal{C} \cup \overline{R_{t_{1}}} \cup \cdots \cup \overline{R_{t_{k}}}, \\
\mathcal{C}_{1}=P^{-1}\left(\mathcal{C}_{0}\right) .
\end{gathered}
$$

Now let $U_{0}$ (resp. $U_{1}$ ) be the unique component of the complement of $\mathcal{C}_{0}$ (resp. $\mathcal{C}_{1}$ ) such that $\hat{z}$ lies in the boundary of $U_{0}$ (resp. $U_{1}$ ). The same arguments used before show that $U_{0}$ contains at most finitely many cycles. Since the union of $U_{0}$ and a preimage of $\overline{R_{t_{0}}}$ is an open neighborhood of $\hat{z}$, it follows that $\hat{z}$ cannot be approximated by small cycles.

Theorem 1.1 Let $P$ be a polynomial with a Cremer fixed point $\hat{z}$ that is approximated by small cycles. Then there exists a critical point which is not accessible from $\mathbb{C} \backslash J_{P}$.

For the case in which $J_{P}$ is connected, Theorem 1.1 is an immediate consequence of Theorem 1.3. In the case that $J_{P}$ is disconnected the idea is to extract from $P$ a polynomial like map which after straightening becomes a Cremer polynomial with connected Julia set. Then we can apply Theorem 1.3 to obtain a non-accessible critical point. Before going further we need some definitions and results about polynomial like mappings (compare [DH]):

Definition: Let $D \subset \mathbb{C}$ and $D^{\prime} \subset \mathbb{C}$ be bounded simply connected domains with smooth boundaries such that $\bar{D} \subset D^{\prime}$. The triple $\left(f ; D, D^{\prime}\right)$ is called a polynomial like map of degree $d$, if the map $f: \bar{D} \rightarrow \overline{D^{\prime}}$ is a $d$-fold branched covering $(d \geq 2)$ which is holomorphic in $D$.

The filled Julia set $K_{\left(f ; D, D^{\prime}\right)}$ of $\left(f ; D, D^{\prime}\right)$ is the set of points in $D$ for which the forward iterates of $f$ are well defined:

$$
K_{\left(f ; D, D^{\prime}\right)}=\bigcap f^{-n}(\bar{D}) .
$$

The Julia set $J_{\left(f ; D, D^{\prime}\right)}$ is the boundary of $K_{\left(f ; D, D^{\prime}\right)}$.

Polynomial like mappings can be extended to the complex plane in such a way that they are quasiconformally conjugate to a polynomial of the same degree:

Theorem 4.1 (Douady and Hubbard) If $\left(f ; D, D^{\prime}\right)$ is a polynomial like map of degree $d$, then there exists a quasiconformal map $\phi: \mathbb{C} \rightarrow \mathbb{C}$ and a polynomial $Q$ of degree $d$ such that $\phi \circ f=Q \circ \phi$ on $\bar{D}$. Moreover, $\phi\left(K_{\left(f ; D, D^{\prime}\right)}\right)=K_{Q}$. 
Now we have all the ingredients to prove Theorem 1.1 except for the following:

Lemma 4.2 Let $P$ be a polynomial and $C$ a connected component of $K_{P}$ such that $P(C)=C$. If $C$ is not a repelling fixed point then there exists a polynomial like map $\left(P ; D, D^{\prime}\right)$ such that $C=K_{\left(P ; D, D^{\prime}\right)}$.

Let us defer the proof of this Lemma.

Proof of Theorem 1.1: Let $C$ be the connected component of $K_{P}$ that contains the Cremer fixed point $\hat{z}$. It follows that $P(C)=C$. Extract a polynomial like map $\left(P ; D, D^{\prime}\right)$ as in Lemma 4.2. After 'straightening' $\left(P ; D, D^{\prime}\right)$ we obtain a polynomial $Q$ and a homeomorphism $\phi$ such that $\phi \circ f=Q \circ \phi$ on $\bar{D}$ and $K_{Q}=\phi(C)$ (Theorem 4.1). Notice that $K_{Q}$ is connected. Hence, $J_{Q}=\phi(\partial C)$ is connected.

Every neighborhood $U \subset D$ of $\hat{z}$ contains infinitely many cycles of $P$, hence every neighborhood of $\phi(\hat{z})$ contains infinitely many cycles of $Q$. Therefore, $\phi(\hat{z})$ is a Cremer fixed point of $Q$ that is approximated by small cycles. Now we are under the assumptions of Theorem 1.3 for connected Julia sets, let $c \in J_{Q}$ be a critical point of $Q$ that is not accessible from $\mathbb{C}-J_{Q}$.

It is not difficult to check that $\phi^{-1}(c)$ is critical point of $P$ that belongs to $\partial C \subset J_{P}$. Also, paths in $\mathbb{C} \backslash J_{Q}$ correspond under $\phi^{-1}$ to paths in $\mathbb{C} \backslash \partial C$. Thus, the critical point $\phi^{-1}(c)$ of $P$ is not accessible from $\mathbb{C} \backslash \partial C \supset \mathbb{C} \backslash J_{P}$. 
In order to prove Lemma 4.2 we need to work with the Green function $G$ of $K_{P}$ :

$$
\begin{aligned}
G: \mathbb{C} & \rightarrow \mathbb{R}_{\geq 0} \\
z & \mapsto \lim _{n \rightarrow \infty} \frac{\log _{+}\left|P^{\circ n}(z)\right|}{d^{n}}
\end{aligned}
$$

where $\log _{+} s=\max \{0, \log s\}$.

It can be shown that $G$ is continuous, vanishes in $K_{P}$ and $G \circ P=d \cdot G$. The Green function is harmonic in $\mathbb{C}-K_{P}$, in particular differentiable. If $r>0$ is a regular value of $G$, then each component of $\{z: G(z) \leq r\}$ is a closed topological disc with smooth boundary. A point $z \in \mathbb{C} \backslash K_{P}$ is a critical point of $G$ if and only if some forward iterate of $z$ is a critical point of $P$. Thus, $G$ has regular values arbitrarily close to 0 . (see Section 17 of [M1])

Proof of Lemma 4.2: Let us denote by $V(r)$ the connected component of $\{z: G(z) \leq r\}$ containing $C$. It is not difficult to check that:

$$
C=\bigcap V(r)
$$

where the intersection is taken over all the regular values $r$ of $G$.

In particular, if a critical point $c$ of $P$ is such that $c \in V(r)$ for all regular values $r$ of $G$, then $c \in C$. It follows that there exists a regular value $r_{0}$ of $G$ such that all the critical points of $P$ contained in $V\left(r_{0}\right)$ belong to $C$.

Consider the topological discs with smooth boundary $D^{\prime}=\operatorname{int} V\left(r_{0}\right)$ and $D=\operatorname{int} V\left(r_{0} / d\right)$. The proper holomorphic map $P: D \rightarrow D^{\prime}$ is a $d$-fold branched covering. Under the assumption that $C$ is not a repelling fixed point, $\left.P\right|_{D}$ is not a conformal isomorphism. Hence, $d \geq 2$ and $\left(P ; D, D^{\prime}\right)$ is a polynomial like map.

The critical points of the polynomial like map $\left(P ; D, D^{\prime}\right)$ are contained in $C$, therefore they never escape from $D$. It follows that the filled Julia set $K_{\left(P ; D, D^{\prime}\right)}$ is connected and $C \subset K_{\left(P ; D, D^{\prime}\right)} \subset K_{P}$. But $C$ is a connected component of $K_{P}$, so in fact $C=K_{\left(P ; D, D^{\prime}\right)}$.

Corollary 1.2 The critical point of a quadratic polynomial $P$ with a Cremer periodic point $\hat{z}$ is not accessible from the complement of the Julia set.

The proof of Corollary 1.2 consists in showing that Cremer periodic points of quadratic polynomials are aproximated by small cycles. This is obtained by putting together results of Douady, Hubbard and Yoccoz. According to Douady and Hubbard a quadratic polynomial $P$ with a Cremer periodic point (not fixed) is renormalizable. After an appropiate renormalization of $P$ we obtain a quadratic polynomial with a Cremer fixed point which, according to Yoccoz, is approximated by small cycles:

Proof: Let $n$ be the period of $\hat{z}$. It follows from the work of Douady and Hubbard [DH] that there exists a quadratic like map $\left(P^{\circ n}, D, D^{\prime}\right)$ which after straightening becomes a quadratic polynomial $Q$ with a Cremer fixed point. More precisely, call $\phi$ the quasiconformal conjugacy between $\left(P^{\circ n}, D, D^{\prime}\right)$ and $Q$ (Theorem 4.1), then $\phi(\hat{z})$ is a Cremer fixed point of $Q$. According to Yoccoz [Y] $\phi(\hat{z})$ is approximated by small cycles of $Q$. Therefore, $\hat{z}$ is approximated by small cycles of $P^{\circ}$. Now apply Theorem 1.1 to $P^{\circ n}$ and obtain a non-accessible critical point of $P^{\circ n}$. Since the critical points of $P^{\circ n}$ are preimages of the critical point of $P$ and accessibility is a completely invariant property, it follows that the critical point of $P$ is not accessible from the complement of the Julia set. 


\section{References}

[BL] B. Bielefeld and M. Lyubich(editors), Problems in Holomorphic Dynamics, In: Linear and Complex Analysis Problem Book 3, vol III, (eds. V. P. Havin and N. K. Nikolskii), Springer 1994. [IMS preprints 1992/7].

[Bl] P. Blanchard, Complex analytic dynamics on the Riemann sphere, Bull. Amer. Math. Soc. 11 (1984), 85-141.

[Cr] H. Cremer, Zum Zentrumproblem, Math. Ann. 98 (1927) 151-163.

[DH] A. Douady and J. H. Hubbard, On the dynamics of polynomial-like mappings, Ann. Sci. Ec. Norm. Sup. $4^{e}$ série 18 (1985), 287-343.

[EL] A. E. Eremenko and G. M. Levin, Periodic points of polynomials, Ukranian Math. J. 1 (1990), 563-633.

[GM] L.Goldberg and J.Milnor, Fixed points of polynomial maps, Part II. Fixed point portraits, Ann. Sci. Ec. Norm. Sup. $4^{e}$ série 26 (1993), 51-98.

[H] J.H. Hubbard. Local connectivity of Julia sets and bifurcation loci: three theorems of J.-C. Yоссоz. pp 467-511 of "Topological Methods in Modern Mathematics", Publish or Perish, 1993.

[M1] J. Milnor, Dynamics in one complex variable: Introductory lectures, Stony Brook IMS Preprint 1990/5.

[M2] J. Milnor, New version of Section 8 of [M1].

[L] M. Lyubich, The dynamics of rational transforms: the topological picture, Russian Math. Surveys 41:4 (1986), 43-117.

[PM1] R. Pérez-Marco, Solution complete au probleme de Siegel de linearisation d'une application holomorphe au voisinage d'un point fixe (d'apres J.-C. Yoccoz), Sém. Bourbaki 753, Feb. 1992, published in Astérisque 1993.

[PM2] R. Pérez-Marco, Topology of Julia sets and hedgehogs, preprint Université Paris-Sud, 1994.

[Ru] W. Rudin, Real and Complex Analysis, McGraw-Hill, 1987.

[Si] C.L. Siegel, Iteration of analytic functions, Ann. of Math. 43 (1942) 607-612.

[Sh] M. Shishikura, On quasiconformal surgery of rational functions, Ann. Sci. Ec. Norm. Sup. $4^{e}$ série 20 (1987), 1-29.

[Sø] D. E. K. Sørensen, Local connectivity of quadratic Julia sets, preprint, Tech. Univ. Denmark, Lyngby 1992.

[S1] D. Sullivan, Conformal dynamical systems pp. 725-752 of "Geometric Dynamics", edit. J. Palis, Lect. Notes Math. 1007 , Springer 1983.

[S2] D. Sullivan, Quasiconformal homeomorphism and dynamics I, solution of the Fatou-Julia problem on wandering domains, Ann. Math. 122 (1985) 401-418. 
[Y] J.-C. Yoccoz, Théorème de Siegel, polynômes quadratiques et nombres de Brjuno, preprint (1987). 